\title{
Apuntes para una intervención participativa y comunitaria en contextos de diversidad cultural
}

\author{
Notes on a participatory anad community intervention \\ within the context of cultural diversity \\ Manuel BASAGOITI RODRÍGUEZ \\ Universidad Carlos III de Madrid \\ mbasagoi@polsoc.uc3m.es \\ Paloma BRU MARTín \\ Universidad Carlos III de Madrid \\ mbru@polsoc.uc3m.es
}

Recibido: 09/02/2012

Revisado: 29/02/2012

Aceptado: 03/04/2012

Disponible on line: 08/03/2012

\section{Resumen}

El fenómeno de las migraciones ha convertido la diversidad cultural en un rasgo fundamental de nuestras sociedades. Sin embargo, esta nueva realidad, palpable en los cambios demográficos de muchas ciudades y en el aumento de necesidades sociales, no se ha visto acompañada de políticas sociales ágiles y adecuadas. Así, la incorporación social de las personas de origen extranjero a nuestro país no ha sido fácil y como consecuencia, su participación como ciudadanos y ciudadanas tampoco.

Partiendo de una contextualización general sobre la evolución de los movimientos migratorios y el reflejo que tiene el aumento de la diversidad cultural en nuestra sociedad en el enfoque de las políticas públicas de integración, en este artículo queremos apuntar (como versa el título) algunas reflexiones sobre la importancia de los contextos locales y de la participación social en la incorporación de la población inmigrante y, a partir de experiencias de trabajo en diferentes barrios de Madrid, proponer algunas pistas o líneas de trabajo para construir modelos de intervención participativa en contextos de diversidad.

Palabras clave: integración social, participación social, ciudadanía, programas comunitarios, inmigración.

\begin{abstract}
The phenomenon of migration has made cultural diversity a fundamental feature of our societies. This new reality, however, evident in the demographic changes in many cities and in the increase of social needs, has not been accompanied by adequate and agile social policies. So, social integration of immigrants in our country has not been easy and, consequently, neither has civic participation.

Based on a general contextualization of the evolution of migration and the reflection that increasing cultural diversity in our society has on the public policy of integration, we note in this article (as suggested by the title) some reflections on the importance of local contexts and social participation in the integration of immigrants and, based on work experiences in different areas of Madrid, propose some clues or leads on work to build participatory intervention models in diverse contexts.
\end{abstract}

Key words: social integration, social participation, citizenship, community work, immigration.

Referencia normalizada: Basagoiti Rodríguez, Manuel, y Bru Martín, Paloma (2012); «Apuntes para una intervención participativa y comunitaria en contextos de diversidad cultural». Cuadernos de Trabajo Social, 25(2): 371-381.

Sumario: 1. Los procesos migratorios en España. Las razones de la emigración ayer y hoy. 2. Políticas públicas de integración de los inmigrantes y gestión de la diversidad. 3. Intervención participativa y comunitaria con los inmigrantes. 4. Algunas conclusiones y propuestas. 5. Referencias bibliográficas. 


\section{Los procesos migratorios en España. Las razones de la emigración ayer y hoy}

A lo largo de su historia, España se ha visto afectada por diferentes procesos migratorios, de españoles hacia otros países y de personas de fuera hacia nuestro país. Haciendo un breve repaso por la memoria histórica de la migración española, desde el inicio de la Guerra civil hasta nuestros días, podemos destacar las siguientes tendencias en los flujos migratorios:

\subsection{De dentro afuera}

- La emigración hacia América Latina. A partir del año 1936 y durante la posguerra y la dictadura franquista el flujo de exiliados españoles se dirigió fundamentalmente a México.

- La emigración hacia Europa: Entre 1950 a 1970, en plena dictadura franquista, los flujos de emigrantes económicos se dirigieron a Alemania, Francia y Suiza.

- La migración interior del campo a la ciudad. También en las décadas de los 1950 a 1970 el llamado éxodo rural movilizó a grandes contingentes de agricultores del interior y sur peninsular hacia las grandes ciudades españolas, Madrid, Barcelona y Bilbao.

- España, de nuevo país de emigrantes. Desde 2010, a consecuencia de la crisis y el crecimiento del desempleo juvenil, se ha incrementado el volumen de españoles que deciden salir de nuestro país a trabajar y residir en otros lugares.

\subsection{De afuera adentro}

Desde finales de los años 1970 y durante el primer lustro del siglo XXI, Madrid, al igual que el resto de comunidades autónomas experimentó la llegada de población de otros países. En estos procesos migratorios globales podemos distinguir dos etapas, una moderada que podríamos encuadrar entre 1975 y 1995 y una intensa de 1995 a la actualidad. En la primera etapa los flujos provienen fundamentalmente de África, Asia y finalmente Latinoamérica. Posteriormente se intensifican desde Latinoamérica y Europa del Este.

En estos y otros procesos migratorios se combinan elementos expulsores de los países de origen (crisis económica, corrupción o persecución política, guerra, desastres medioambientales, altos índices de desigualdad y pobreza en entornos de riqueza en recursos naturales que no se redistribuyen, deslocalización de las empresas y privatización de los bienes y servicios públicos que ponen los recursos en manos de multinacionales extranjeras, incertidumbres sobre el futuro y el desarrollo de dichos países, violencia institucionalizada, etc.) y elementos de atracción hacia otros estados o continentes (mayor desarrollo y estabilidad política, económica, jurídica y social, demanda de mano de obra poco cualificada, imagen de riqueza y prosperidad, existencia de familiares y compatriotas en otros países, etc.).

Este «efecto llamada» se ha visto frenado desde el año 2008 con motivo de la crisis. Se reduce drásticamente la llegada de población inmigrante por el aumento del desempleo en España y Europa y se inician movimientos de retorno a los países de origen de los inmigrantes, forzados por el incremento de expulsiones de inmigrantes en situación irregular e impulsados, en menor medida, por el refuerzo de políticas de apoyo al retorno.

Podemos considerar el proyecto migratorio como una «trayectoria vital» llena de altibajos, con avances y retrocesos, en la que inciden multiplicidad de factores comunes y específicos, y donde la fuerza individual y las condiciones del entorno tienen una influencia determinante.

La llegada al país de acogida es una primera fase que comporta una crisis importante. $\mathrm{Al}$ surgir de una separación de la tierra, de la familia, etc. implica un alto coste emocional y psicológico y necesita de mayores apoyos externos. Esta ruptura dolorosa va acompañada de conflicto, desarraigo, desorientación, necesidad de ingresos, vulnerabilidad social, etc., pero también de nuevas expectativas y relaciones, muchas veces posibilitadas por profesionales y centros de acogida.

El asentamiento y la decisión de quedarse es un segundo momento en el que se produce la adaptación al nuevo contexto sociocultural, y suele coincidir con la consolidación de redes propias de apoyo, el inicio de procesos de regularización, se abre el objetivo de la reagrupación familiar, se busca información de recursos que se ajusten a sus necesidades y respondan a sus expectativas (ayudas, formación, acompañamiento, etc.)

La consolidación de esta trayectoria se produce con la mejora de las condiciones de trabajo, el acceso a recursos, prestaciones sociales y 
derechos laborales (en el caso de los inmigrantes económicos) y la posibilidad real de un desarrollo y promoción personal, profesional y social. Este momento tiene efectos favorables tanto para el inmigrante y su entorno en España como para su familia de origen (aumenta su capacidad de retornar bienes). Este momento es en el que se puede encontrar una mayor participación por parte de las personas inmigrantes. La resolución de los problemas y conflictos de la llegada y el asentamiento en nuestro país implican seguridad y una mayor disposición a participar en los entornos más cercanos.

Finalmente, tras la consolidación se comienzan a dar pasos hacia la integración, aunque para que esta sea plena es necesaria la incorporación a la sociedad receptora en igualdad de condiciones, derechos, obligaciones y oportunidades que los ciudadanos autóctonos, la preservación de la cultura de origen y la aceptación por parte de la mayoría social de los cambios normativos, institucionales e ideológicos necesarios para que lo anterior sea posible, condiciones que se ven frenadas por el contexto político europeo actual.

No obstante, en esta nueva etapa, el imaginario social de parte de la población inmigrante interioriza esta integración en acciones que implican «la decisión de quedarse» y hacer vida en España, como la reagrupación familiar, los nuevos matrimonios mixtos, tener más hijos, solicitar la nueva nacionalidad, etc. Una segunda y tercera generaciones de familias inmigrantes van teniendo cada vez más presencia y asientan aún más su estatuto de nuevos vecinos $\mathrm{y}$ vecinas. Por ello, es en estas últimas etapas cuando los procesos de participación ciudadana y trabajo comunitario encuentran un contexto más favorable.

\section{Políticas públicas de integración de los inmigrantes y gestión de la diversidad}

En España, los múltiples procesos de regularización a los que nos hemos enfrentado desde el año 1985 (1985, 1991, 1996, 2001, 2003 y 2005) revelan un modelo inestable y desordenado, que ha traído consecuencias visibles en el asentamiento de la población inmigrante no sólo a escala nacional, sino también en nuestras ciudades y nuestros barrios.

Vivimos tiempos en los que las dinámicas sociales se adelantan permanentemente a las políticas públicas y desvelan, cada vez con mayor crudeza, las desigualdades sociales. Muchos de los síntomas de estas desigualdades los encontramos en ciudades y distritos. En estos años hemos visto aumentar la población de nuestros barrios y las situaciones de vulnerabilidad, pero los recursos públicos apenas han aumentado y muchos de ellos han sido suprimidos: en Madrid, los Centros de Apoyo Social a Inmigrantes, el Observatorio de las Migraciones, el servicio de Mediación Social Intercultural, etc., víctimas de los recortes sociales (programas de integración desarrollados por muchas Ongs) o privatizados. Las políticas de integración y protección social, fomento del empleo, promoción de la educación y la sanidad pública y de calidad, etc. se enfrentan a vientos muy poco favorables.

El reto de la diversidad, si podemos llamarlo así, se encuentra actualmente en el centro mismo del debate político, social y económico. Se plantea como gestión de la diversidad, cuando aún falta por descubrir qué se esconde detrás de estas palabras. Diversidad sí, pero gestión, ¿cómo? En España no existe un modelo coherente que haya sabido abordar estas cuestiones.

El crecimiento vertiginoso de la llegada de inmigrantes, la existencia de un proceso creciente interno de debate sobre los derechos de pluralidad nacional y regional, la puesta en marcha de muchas acciones pero sin coordinar y sin una coherencia global: iniciativas regionales y locales, planes de inmigración, observatorios regionales, etc. pero cada una desde una lógica diferente (...). Son algunas razones que han ido dando como resultado un entramado normativo poco integrado y poco coordinado que se mueve entre modelos que fomentan una integración de la población inmigrante a partir de una actuación más institucional, hasta modelos que confían más en la autorregulación de los mercados (de empleo, de vivienda, matrimoniales...) (VV.AA., 2008).

Este debate parece seguir enquistado entre dos modelos de referencia: el multiculturalista anglosajón y el asimilacionista francés. Ninguno de los dos modelos ha favorecido la participación plena de la población inmigrante en nuestras sociedades; el primero, siendo un gran defensor de la igualdad de derechos y oportunidades y del respeto a la diferencia cultural, no se ha mostrado eficaz en la gestión de la in- 
tegración, dando lugar a espacios relacionales en los que la coexistencia prima sobre la convivencia, surgiendo incluso guetos que segregan a los diferentes grupos sociales; el segundo, al apostar por una integración cuyo eje principal es la asimilación de la cultura y las normas del país de acogida, ha traído como consecuencia la desconfianza, el desarraigo y la aculturación de muchas personas extranjeras, que sienten que están bajo permanente sospecha.

Por otro lado, la actual crisis económica da alas a planteamientos políticos (y sociales) más segregacionistas y xenófobos, que endurecen los controles fronterizos, dificultan la reunificación familiar, restringen el acceso a los servicios básicos, aumentan la represión de los inmigrantes, etc.

El humo de hace casi tres años de los campamentos gitanos que ardieron en Italia nos puso sobre aviso de lo que se avecinaba. Desde entonces, elección tras elección, los xenófobos han ido ganando fuerza en nuevos países (Suecia, Finlandia, Reino Unido, Hungría) y consolidándose donde ya tenían presencia importante (Italia, Francia, Dinamarca) (Torreblanca, 2011).

Este proceso también afecta a España, puerta de entrada de la inmigración africana, que se ve presionada para seguir estas directrices. En nuestro país, en las últimas décadas se han ido definiendo diferentes políticas de regulación de los flujos migratorios, desde el control de fronteras contra la inmigración irregular al establecimiento de cuotas para cubrir empleos en España con gente de fuera (cubiertos en su mayoría con inmigrantes irregulares que ya estaban en España), acuerdos bilaterales con algunos países como Marruecos, Colombia, Ecuador, Rumania, República Dominicana, Polonia, Bolivia, etc. Sin embargo, la idoneidad de muchas de estas medidas es bastante discutible y su eficacia limitada. Frente a esta realidad, la reforma de la Ley Orgánica 4/2000, de 11 de enero, sobre derechos y libertades de los extranjeros y su integración social, recogida en el objetivo 4 de la Ley Orgánica 2/2009, de 11 de diciembre, plantea:

Reforzar la integración como uno de los ejes centrales de la política de inmigración que, teniendo en cuenta el acervo de la Unión Europea en materia de inmigración y protección internacional, apuesta por lograr un marco de convivencia de identidades y culturas.

Su desarrollo, a través de Planes de Integración para población inmigrante, permitiría: «el paso de un nivel satisfactorio de coexistencia (multiculturalismo) - una coincidencia en tiempo y espacio de carácter pacífico- a un nivel óptimo de convivencia, la cual supone la interacción y relación armoniosas (interculturalismo)». En este sentido, en el texto del I y II Plan Estratégico de Ciudadanía e Integración (20062010 y 2011-2014), se plantean tres principios políticos que orientarán todas las actuaciones: el principio de igualdad y no discriminación, que implica la equiparación de derechos y obligaciones de la población inmigrante a la autóctona, dentro del marco de los valores constitucionales básicos; en el principio de ciudadanía, que implica el reconocimiento de la plena participación cívica, social, económica, cultural y política de los ciudadanos y ciudadanas inmigrantes; y por último, el principio de interculturalidad, como mecanismo de interacción entre las personas de distintos orígenes y culturas, dentro de la valoración y el respeto de la diversidad cultural.

Paradójicamente, esta declaración de buenas intenciones choca con la pervivencia de instituciones como los Centros de Internamiento para inmigrantes (CIES), denunciados en diferentes ocasiones por las organizaciones de defensa de los derechos humanos, y con la realidad de la aplicación de estos planes en lo local, ya que la diversidad de voluntades políticas existentes en las diferentes comunidades autónomas hace que la implantación de estos principios sea más bien escasa, imperando aún los programas de integración y convivencia diseñados desde lógicas marcadamente asimiladoras.

La integración es entre otras cosas un proceso de equiparación de derechos, y cualquier violación de los mismos conlleva situaciones de vulnerabilidad e injusticia social que fracturan la cohesión social. La limitación de derechos de los ciudadanos extranjeros comporta un efecto perverso en las relaciones de convivencia entre la población de origen extranjera y la autóctona, en los contextos micro locales de interacción social y en los equipamientos de 
proximidad (barrio, escuela, centro de salud, Servicios Sociales, etc.). Evitar la competencia entre la población inmigrante y la española por unos recursos cada vez más limitados, obliga a reforzar los servicios básicos como son la sanidad, la educación y la protección social, para evitar que éstos pierdan calidad para todos.

En esta labor, el Tercer Sector también tiene que desempeñar un papel importante. Desde la aparición de las primeras organizaciones de atención a población inmigrante en 1985 hasta la actualidad ha crecido y no ha dejado de desarrollar iniciativas sociales, en plataformas y redes que buscaban una respuesta a esa complejidad, casa vez mayor, de los problemas sociales. En estos años ha asumido funciones claves en lo que se refiere a la gestión de la diversidad, la convivencia y la integración de personas inmigrantes: en la detección de sus necesidades, facilitada por su cercanía y proximidad a estos colectivos; en la provisión de servicios sociales que no cubre la Administración (apoyo al empleo, emergencia y supervivencia, cobertura de necesidades sociales, como vivienda, educación, empleo, etc., o gestiones administrativas, económicas, etc.); en el fomento del asociacionismo y la participación social; en la orientación específica a las poblaciones más vulnerables; en la promoción de valores de solidaridad y la reivindicación sobre derechos sociales, etc.

\section{Intervención participativa y comunitaria con los inmigrantes}

\subsection{Procesos de participación de los inmi- grantes}

En la Comunidad de Madrid podemos diferenciar dos tipos de procesos que aspiran a otorgar un mayor protagonismo a las personas de origen extranjero que residen en ella.

Existen por un lado procesos institucionalizados $-\mathrm{o}$ instituidos formalmente «de arriba abajo»- como son las Mesas de Convivencia del Ayuntamiento de Madrid, el Foro Madrid de Diálogo y Convivencia, los Planes de Convivencia distritales o los Centros de Participación e Integración de la Comunidad de Madrid (CEPI). Estos instrumentos conviven con los procesos participativos y las redes locales comunitarias, iniciativas constituidas «de abajo arriba», desde el tejido social local — asociaciones, sectores funcionalmente proactivos, grupos in- formales, etc. - que tienen como objetivo el desarrollo y la mejora de la calidad de vida a través de la participación de todos los agentes sociales presentes en el territorio. Algunos ejemplos los podemos encontrar en Carabanchel, Tetuán, Villaverde y Usera, Vallecas, etc.

Se entiende que las posibilidades de convivencia como el desarrollo de relaciones vecinales entre diferentes grupos - autóctonos y extranjeros - en las que rige el respeto mutuo y el diálogo en igualdad, son difíciles, pero lo son aún más según la actitud que manifiesten y mantengan los propios vecinos ante la misma. En este sentido creemos que las entidades sociales de arraigo territorial y los profesionales y sectores funcionalmente activos de la Administración con presencia activa en el territorio (trabajadores sociales comunitarios, educadores, mediadores, dinamizadores, etc.) tienen una tarea importante y la responsabilidad de fomentar espacios donde el diálogo sea posible y donde se pueda construir un modelo común de convivencia.

La participación ciudadana constituye el motor del desarrollo local comunitario, y entendemos que esta estrategia de acción colectiva organizada puede muy bien articularse a través de las entidades sociales con arraigo en la comunidad y capacidad de caminar «de la mano» con otros agentes institucionales, sociales, grupos y organizaciones que también contribuyen a «hacer barrio y ciudad», como las parroquias, las AMPAS, las ONG, las asociaciones culturales, deportivas, de ayuda mutua, ecologistas y las coordinadoras y plataformas). La participación no puede ejercerse de la forma individualista, atomizada y aislada en que se hallan muchas entidades sociales en los barrios y distritos de Madrid, porque le resta eficacia e impacto a la acción.

Estas iniciativas de la ciudadanía organizada y las entidades sociales abordan los problemas locales desde la diversidad cultural y social, avanzando de forma colectiva hacia modelos de desarrollo territorial y comunitario con las bases de la participación ciudadana y del trabajo en red. Ese nuevo paradigma relacional que se basa en la cooperación requiere iniciativa política, innovación social y consenso ciudadano, y plantea en definitiva el reto de superar el desencuentro secular entre el sistema institucional y las entidades ciudadanas. 


\subsection{Los entornos de acogida. Relevancia de lo local y barrial}

La llegada de inmigrantes ha ido creciendo exponencial hasta el año 2009, ha pasado de cifras de algo más de 50.000 extranjeros empadronados en 1996 en el Municipio de Madrid a 574.869 en 2009, año en el que se ha puesto de manifiesto una tendencia inversa hacia su decrecimiento: en enero de 2012 la presencia de inmigrantes había descendido a 502.932 personas.

Los barrios a los que se han ido incorporando han sido aquellos con el precio de la vivienda más barato (por su escasa habitabilidad, lejanía, aislamiento, etc.) y en muchas ocasiones donde se acumulan más problemas urbanos y sociales. Así, los nuevos vecinos se iban asentando en contextos donde deben cohabitar con sectores poblacionales muy diferentes entre sí, pero que comparten factores comunes de desfavorecimiento y vulnerabilidad: desempleo o empleo precario, bajo nivel educativo y formativo, dependencia de prestaciones sociales, vivienda precaria, escasez de equipamientos, de espacios de ocio y al aire libre, situaciones de desestructuración familiar, violencia doméstica y otras patologías, etc.

La llegada de la inmigración ha tenido sin duda efectos positivos en Madrid. Ha supuesto una fuerte revitalización de estos barrios en diferentes aspectos y dimensiones de desarrollo, pues las familias, sean de origen autóctono o foráneo, constituyen una unidad de producción, consumo y reproducción social, con todo lo que ello conlleva de impulso y crecimiento económico, social, cultural, etc. Cabe apuntar hasta el año 2008 el crecimiento sostenido del PIB, el superávit en la Seguridad Social, el aumento de las tasas de natalidad, el rejuvenecimiento poblacional, etc. Por otro lado, el aumento de población provocado por la inmigración implica un uso más intensivo de servicios y recursos públicos, por sí mismos ya bastante insuficientes. Este aspecto, unido a lo que muchos llaman una crisis del Estado del Bienestar, da argumentos a discursos de marcado corte racista y xenófobo, para situar a los inmigrantes en el disparadero en la carrera por la búsqueda de culpables de esta crisis.

Desde el año 2008 hasta la actualidad, en el contexto de la crisis económica, la contención a la llegada de población inmigrante y el aumento de población española que emigra hacia otros países parece poner en grave peligro este fondo de sostenimiento social, al que contribuyeron a mantener a flote muchos de los extranjeros que ahora son considerados innecesarios y en muchos casos expulsados de nuestro país.

Como ya hemos señalado, los barrios a los que se incorporan las personas inmigrantes tienen características de vulnerabilidad urbana y social, pero también son espacios en los que es posible establecer relaciones sociales comunitarias, que cumplen un papel afectivo, de protección y apoyo o información fundamental y se convierten en un marco multicultural y potencial para el desarrollo de la convivencia en la diferencia. Así, los entornos microlocales (calle, barrio, distrito, pueblo) constituyen un nivel de actuación factible, donde pueden tomar forma: la promoción de redes sociales comunitarias, la concertación entre diferentes tipos de agentes sociales y la promoción de la participación activa y comprometida.

La interacción social próxima, «cara a cara», facilita el compromiso emocional con el «otro» (el vecino, trabajador como nosotros, y también padre, hermano, hijo); con quien se comparten experiencias comunes de carencias y necesidades, y también ayuda a comprender que es más lo que nos une que lo que nos separa o diferencia. En esta interacción va creciendo el sentido de identidad comunitaria, que contribuye a la mejora y la calidez de nuestras relaciones con los otros, sean vecinos, miembros de una misma comunidad, conciudadanos o creyentes de una misma religión. El sentimiento de identidad es fuente de orgullo y alegría, fuerza y confianza. Pero la identidad también puede ser fuente de conflicto, cuando este sentimiento de pertenencia «fuerte» se construye en oposición a la de otros grupos sociales; es la identidad excluyente o auto-excluyente que conforma una suerte de solidaridad interna y una distancia o divergencia insalvable de los otros grupos.

Los problemas de convivencia no pueden abordarse únicamente con parámetros de conflicto de identidad cultural, sino como problemas de integración que tienen en su raíz situaciones carenciales que afectan al bienestar de individuos, familias y barrios que conviven y compiten por los mismos bienes. Y tales conflictos han de resolverse a través de la participación ciudadana, entendida como «acción co- 
lectivamente organizada bajo principios de solidaridad y bienestar común».

En nuestro punto de vista, la construcción de identidades comunitarias y la participación social están íntimamente relacionadas. De acuerdo con García Roca (2004), la participación responde a una doble dinámica: ser parte (quedar vinculado) y tomar parte (elegir la pertenencia); sin vinculación no hay participación social, pero sin opción personal tampoco hay participación humana. La doble dinámica de la participación - involuntaria y voluntaria- quiere subrayar que la vida humana no está hecha sólo de asociaciones a las que pertenecemos involuntariamente (familia, nación, clase, partidos políticos...) y que habitualmente heredamos (aunque incluso en estos casos queda evidentemente la posibilidad de aceptarlos o desvincularnos); sino que existe la posibilidad de establecer asociaciones y, de este modo, la participación preserva y aumenta la libertad frente al determinismo, recupera la voluntad frente a lo forzoso de lo dado, reivindica la innovación frente al mecanicismo.

\section{3. ¿Cómo desarrollamos procesos de par- ticipación comunitaria?}

Nuestra propuesta metodológica contempla la construcción colectiva de espacios comunitarios en los que sea posible la confluencia de los diferentes grupos sociales formales e informales y agentes técnicos e institucionales en un mismo proceso de intercambio, debate y acción social. Este tipo de procesos comunitarios debe estar encaminado a la transformación y mejora de las condiciones económicas, sociales y culturales del conjunto de la comunidad local, partiendo de las necesidades y problemáticas comunes a toda la población y específicas de cada grupo, asentando lazos identitarios que contemplen lo que tenemos de común y lo que nos diferencia. Buscamos alcanzar, de forma colectiva, objetivos comunes de mejora y cohesión social, al formar parte todos de una misma comunidad y tomar parte en un mismo proceso de cambio social.

Para posibilitar la participación de todos los grupos y permitir la creación de oportunidades para su inclusión social, debemos tener en cuenta sus atributos diferenciales. Las mujeres, los mayores, los niños, los jóvenes, los inmigrantes de diferentes procedencias, etc., presentan disponibilidades, intereses, inquietudes, capacidades, potencialidades, que tienen necesariamente que ser acoplados al proceso participativo. Así, en la intervención comunitaria y participativa en contextos de diversidad cultural debemos tener en cuenta cuatro aspectos clave:

En primer lugar, avanzar hacia un conocimiento conjunto de la realidad local. Es decir, analizamos las necesidades y los problemas de forma colectiva y participativa, avanzando hacia un diagnóstico integral comunitario en el que se reconozcan todos los colectivos presentes en el territorio. En este camino debemos tender puentes entre la sociedad emisora y la sociedad receptora, acercándonos a la realidad compleja que hay detrás de cualquier proceso migratorio. No se trata sólo de un viaje con el objetivo de encontrar un empleo, sino de una apuesta vital que concierne a todo un núcleo familiar $y$, por lo tanto, llena de dolor (por lo que se ha dejado atrás) y de esperanza (por las mejoras que se espera encontrar).

En segundo lugar, desarrollamos acciones conjuntas que sirvan para consolidar la participación, dar visibilidad al propio trabajo comunitario y sensibilizar a otros grupos sobre la importancia de este tipo de procesos. Para ello, creamos redes en el territorio en las que puedan participar toda la diversidad de grupos sociales, tanto formales como informales.

En tercer lugar, debemos actuar siempre en clave de proceso, arbitrando actuaciones que respeten los ritmos de los distintos grupos y agentes sociales e institucionales. Así, es importante desarrollar acciones a corto plazo (reuniones, debates, acciones informativas y de comunicación, trabajo de calle, etc.), a medio plazo (organización de acciones de tipo lúdico, formativo, de sensibilización, intervención en problemas específicos, etc.) y a largo plazo (planificación comunitaria, proyectos de intervención en red, iniciativas de creación de empleo o economía social, conexión con experiencias de otros territorios, etc.).

En cuarto lugar, todas estas iniciativas deben tener la diversidad como eje vertebrador y transversal de todo el proceso. Así, contemplaremos la interculturalidad en todas sus etapas.

Un elemento clave es el empoderamiento y acompañamiento de la población, a través de información y formación en la participación y la auto-organización. 


\section{4. ¿De dónde partimos?}

El conocimiento de la realidad es, como decíamos, un obligado punto de partida para el desarrollo comunitario. Conocer aquellos aspectos que le preocupan más a la comunidad es, sin duda, el inicio para cualquier intervención local. Tomando como ejemplo barrios del sur de Madrid, en los que la presencia de población de origen extranjero es alta (San Cristóbal de los Ángeles, Pradolongo, Las Margaritas, San Fermín, Cuatro Caminos, etc.), podemos mencionar algunos aspectos que, de forma recurrente, han suscitado debate y preocupación en la comunidad y que van a influir de forma decisiva en nuestra actuación:

\section{a) Sentimientos de arraigo e identidad de barrio contradictorio}

- El rápido crecimiento de la población inmigrante despierta un cierto temor a la pérdida de la identidad cultural tradicional compartida.

- El cambio demográfico de los barrios también trae consigo modificaciones en las actividades comerciales y de servicios.

- La nueva población aporta enriquecimiento, renovación demográfica y oportunidad de desarrollo para el barrio, tomando cuerpo la identidad en los espacios convivenciales.

- La visibilización de la importancia de la apropiación y el uso de los espacios, recuperados muchas veces por los nuevos vecinos, y de la ausencia de lugares y oportunidades de encuentro y relación en el barrio y la urgente necesidad de construir alternativas para el ocio compartido.

\section{b) Problemas de convivencia intercultural}

- Entre los antiguos vecinos que llevan mucho tiempo en el barrio y los nuevos vecinos, especialmente con población africana y de Europa del Este. En algunos barrios este fenómeno ha ido acompañado por el traslado de la población española hacia otros barrios, en otros de nuevas iniciativas de apoyo a la integración de los nuevos vecinos. Este hecho asienta la idea de ver la diversidad y la convivencia como un problema.

- Surgen conflictos relacionales por: hacinamiento en las viviendas, precariedad laboral, competencia por recursos escasos y lucha por los espacios, etc.
- La población extranjera también se siente rechazada y excluida del conjunto social.

- La visibilidad del cambio en el barrio, especialmente relevante en el uso de los espacios y recursos públicos, generan confusión y miedo, sobre todo entre la población de edad más avanzada.

\section{c) Asentamiento de la vulnerabilidad}

Muchos de estos barrios se han visto tradicionalmente afectados por aspectos como:

- Altas cifras del desempleo respecto a otros barrios y mayor impacto entre jóvenes y mujeres.

- Falta de inversión pública y abandono institucional.

- Percepción de la población de un aumento de la inseguridad y del empeoramiento del barrio.

- Competencia por los recursos entre los diferentes grupos.

- Entre la población inmigrante, sentimiento de exclusión al sentirse rechazada por la población española.

- La percepción de fragilidad o vulnerabilidad social de la comunidad crece y hace necesaria la intervención para prevenir y atajar posibles emergentes procesos de guetización.

- Percepción de la existencia de una pérdida de calidad educativa que se atribuye a la presencia de la población inmigrante y falta de agilidad del sistema educativo para adaptarse a esta nueva realidad.

\section{d) Obstáculos para la participación}

La participación de las personas de origen extranjero es, a todas luces, deficitaria. Algunas razones para ello son:

- Limitación de sus derechos de ciudadanía (civiles, políticos, económicos y sociales), como la imposibilidad del voto. Esta discriminación política es una importante barrera para que los inmigrantes desarrollen un sentimiento de pertenencia y arraigo a nuestra sociedad.

- La participación de los inmigrantes en los órganos de participación municipal (Consejos territoriales, sectoriales...) es excepcional $\mathrm{y}$ las iniciativas que se han intentado poner en marcha en este sentido en los distritos (mesas de diálogo) han fracasado. 
- Escasa disponibilidad de tiempo, debido a la propia emergencia que genera otras prioridades (empleo, familia).

- Desconocimiento de los canales y formas de participación y de la tradición participativa asentada en los barrios.

- La percepción de actitudes de rechazo (en lenguaje, negación de la relación, definición como «el otro», sentimiento de escasa valoración, prejuicios, etc.) genera desconfianza (repliegue en la necesidad individual, búsqueda del propio grupo, refuerzo de la propia identidad, etc.) y también resistencia a la integración a la cultura de acogida (en los dos polos de la relación).

- El miedo a la pérdida de la identidad se da en los dos polos de la relación (por parte de la población española y extranjera), lo que es una clara barrera a la participación.

- Las diferencias culturales, religiosas y de sistema de valores también generan recelos y conflictos, que habitualmente derivan en el asentamiento de prejuicios y estereotipos entre grupos.

- La baja participación no sólo es una realidad que afecta a la población extranjera, ya que en buena parte de la población encontramos muy arraigado el hábito de pensar que los problemas se deben a agentes externos y que otros los deben solucionar.

- El refuerzo del individualismo es una tendencia generalizada que se oculta en ocasiones bajo procesos de identidad colectiva negativa ( definición del otro como culpable, ladrón o delincuente).

- Las asociaciones de inmigrantes son pocas, débiles y con escaso reconocimiento institucional como interlocutores ante las administraciones. Por otro lado, existe una limitada vinculación entre el asociacionismo de inmigrantes (más sectorial, de corte cultural o político) y los procesos de desarrollo local (territorial), salvo en ocasiones por motivaciones religiosas (parroquias, mezquitas), económicas (comercios) y culturales (preservación identidad y tradiciones).

- La percepción por parte de la población española de que los inmigrantes se mueven en redes endogámicas y encapsuladas refuerza la desconfianza y no facilita la construcción de espacios mixtos de participación.

Uno de los retos para la dinamización de la participación es la conexión de lo sectorial con lo local en cuestiones de interés común: derechos humanos, empleo, salud, parques para sus hijos, etc. Las estrategias de acción en el ámbito comunitario deben dirigirse al aumento de la cohesión social como clave para promover la construcción paulatina de una identidad intercultural (identidad de identidades), que se construya de manera dinámica por la interacción de unos individuos con los otros y con los grupos con quienes tejen las relaciones sociales, económicas, políticas.

\subsection{Aspectos que podemos aprovechar pa- ra fomentar la participación y la conviven- cia intercultural}

- La emergencia de cierto asociacionismo y de las redes sociales concienciadas y sensibilizadas. En algunos barrios podemos ver una participación emergente de grupos juveniles o culturales que implica una interacción con el entorno local: grupos de rap, graffiti, skaters, en los centros educativos, asociaciones, etc.

- La cultura en los distritos con un alto índice de diversidad es una oportunidad importante de representación de la convivencia y del encuentro. Desbordar la organización de meras actividades lúdicas y de ocio, mediante otros elementos de organización colectiva y con la diversidad como elemento presente desde que se propone el diseño de las actividades.

- Los conflictos manifiestos, en los que lo fundamental es identificar las razones que los provocan, las relaciones que los hacen surgir y las que se establecen a partir de su estallido (qué alianzas genera, qué rupturas provoca y qué actitudes de apoyo, de indiferencia o de rechazo emergen del mismo). Estas explicaciones pueden evidenciar el germen de nuevas formas de agrupación y organización social.

- Los temas sentidos y focos de interés común. En la convivencia cotidiana podemos buscar cuáles son las inquietudes compartidas (creando comisiones, mesas, plataformas, etc.). En este marco será más fácil favorecer los liderazgos positivos y desbordar los negativos aprovechando la acción colectiva.

- La presencia, directa o indirecta, de los nuevos vecinos en todos los espacios de participación de los barrios, que se puede integrar en la gestión de las asociaciones locales y en todas las actuaciones del fomento de la convivencia y la interculturalidad. 
- Finalmente, la participación festiva, como medio y como fin en sí misma, puede ser el germen de nuevas formas de colaboración y organización ciudadana. En los espacios públicos de los barrios - en plazas, calles, parques, equipamientos culturales, etc.- - se expresan usos e identidades sociales y culturales diferentes, pero también se pueden desplegar acciones conjuntas - de grupos informales (de jóvenes, músicos, artistas, etc.) y de organizaciones sociales (asociaciones de padres, de vecinos, de inmigrantes, juveniles, de tercera edad, etc.) - que hacen visibles y entretejen nuevas relaciones sociales de convivencia.

\section{Algunas conclusiones y propuestas}

Ante la falta de una política de integración clara y definida y de medidas de acompañamiento a los procesos migratorios, las asociaciones, colectivos y sectores funcionalmente proactivos de los barrios están construyendo modelos de paz social «de abajo arriba», en contextos de complejidad y diversidad cultural creciente.

Gran parte del Tercer Sector, sectores funcionales y figuras socioeducativas de los barrios ha empezado a incorporar la perspectiva intercultural en sus actuaciones y a aglutinar, junto a las propuestas habituales, las demandas de la población inmigrante. A través de la promoción de espacios y oportunidades de encuentro y relación, en la fiesta y en el juego, como elementos primarios de «socialidad» - este concepto denota el proceso por el que una sociedad se integra y funciona como tal; esto es, se hace «coherente» y «sólida» o solidaria- es donde se despliegan los vínculos comunitarios que hacen posible que vecinos y grupos de diferente condición —edad, género, etnia- se encuentren y convivan.

Se trata de establecer alianzas estratégicas entre los sectores más dinámicos de la sociedad civil (colectivos autóctonos y de inmigrantes, movimientos sociales, etc.) para impulsar la coordinación, el trabajo en red y el trabajo en equipo desde la innovación, la creatividad, la comunicación y la cooperación, etc.

Muchos de los barrios donde se asientan reúnen características de vulnerabilidad social unidas a la presencia de conflictos sociales y los llamados procesos de participación no terminan de dar el salto de una participación meramente presencial a una comunitaria. Los diferentes grupos, y especialmente las personas inmigrantes no participan en la toma de decisiones de las organizaciones locales, sino que son meros espectadores de la participación, es decir, estamos ante dinámicas en muchas ocasiones asistencialistas, que conciben la participación como un servicio más en busca de usuarios.

Por todo ello, la promoción de estos procesos de participación y desarrollo comunitario debe asentarse en una mayor profundización en estrategias de promoción de la diversidad en un sentido amplio:

- En la acción, combinando la reivindicación con la gestión, la acción en lo local y en lo global, promoviendo iniciativas locales que surjan desde otros grupos informales, apoyándolas, etc.

- En la convivencia, fomentando el acercamiento entre grupos sociales y culturas diferentes.

- En la comunicación, sensibilizando a través de mensajes que transmitan lo positivo de la inmigración, del mestizaje, etc.

- En las concertación social, integrándose en plataformas o redes de actuación territoriales y de dimensión más amplia (regionales, estatales, mundiales, etc.).

- En la participación, promoviendo asambleas y espacios de participación que representen la diversidad cultural y social de los barrios, como puerta hacia la renovación interna de las Asociaciones.

- En la reivindicación, denunciando y visibilizando las consecuencias del mal diseño y aplicación de las políticas en la ciudadanía.

\section{Referencias bibliográficas}

Alguacil, J., Basagoiti, M., Bru, P. y Camacho, J. (2011). Experiencia y Metodología para un Modelo de Desarrollo Comunitario. El caso de San Cristóbal de los Ángeles. Madrid: Editorial Popular.

Arriba, A., Moreno, L. (coords.) (2008). Inmigración, Gestión de la Diversidad y Tercer Sector. Madrid: Fundación Luis Vives.

Basagoiti, M. y Bru, P. (2008). La inmigración, germen y reto del Movimiento Vecinal. En V. Pérez Quintana y P. Sánchez (edits.). Memoria ciudadana y Movimiento Vecinal. Madrid, 19682008. (pp. 336-355). Madrid: Ed. La Catarata. 
Camps, F. (2000). Participación comunitaria y gestión alternativa de conflictos. Cuadernos de Trabajo Social, 15, 231-251.

García Roca, J (2004). Políticas y Programas de Participación Social. Madrid: Síntesis.

Just, J., Martínez, O., Estanyol, V. y Mora, A. (2000). El partenariado como estrategia de trabajo social comunitario. Cuadernos de Trabajo Social, 15, 253-268.

Martínez Aranda, M. A. (2006). Relaciones Vecinales e Inmigración en Madrid.[Monografía] Punto de Vista., 2. Madrid: Observatorio de las Migraciones y de la convivencia intercultural de la ciudad de Madrid. Disponible en: http://www.uam.es/otroscentros/imedes/docs/publi/ omci/monografia_omci_2.pdf

Navarro Pedreño, S. (2000). Contra los puentes levadizos: la formación de trabajadores sociales en clave comunitaria. Cuadernos de Trabajo Social, 15, 183-202.

Secretaria de Estado de Inmigración y emigración (2007). I Plan Estratégico de Ciudadanía e Integración 2007-2010 (PECI). Madrid. Dirección General de Integración de los Inmigrantes.

Secretaria de Estado de Inmigración y emigración (2011). II Plan Estratégico de Ciudadanía e Integración 2011-2014 (PECI). Madrid. Dirección General de Integración de los Inmigrantes. Torreblanca, J.A.( 2011). Cinco razones por las que Europa se resquebraja. El País. 15 de mayo. VV.AA. Movimiento por la convivencia intercultural Sentido Sur (2008). Sentido Sur: Hacia una Ciudadanía Global desde lo Local. Madrid. Edita Asociación San Fermín. 